\title{
AN EFFICIENT METHOD FOR MINIMIZING A CONVEX SEPARABLE LOGARITHMIC FUNCTION SUBJECT TO A CONVEX INEQUALITY CONSTRAINT OR LINEAR EQUALITY CONSTRAINT
}

STEFAN M. STEFANOV

Received 24 May 2005; Accepted 4 October 2005

We consider the problem of minimizing a convex separable logarithmic function over a region defined by a convex inequality constraint or linear equality constraint, and twosided bounds on the variables (box constraints). Such problems are interesting from both theoretical and practical point of view because they arise in some mathematical programming problems as well as in various practical problems such as problems of production planning and scheduling, allocation of resources, decision making, facility location problems, and so forth. Polynomial algorithms are proposed for solving problems of this form and their convergence is proved. Some examples and results of numerical experiments are also presented.

Copyright (c) 2006 Stefan M. Stefanov. This is an open access article distributed under the Creative Commons Attribution License, which permits unrestricted use, distribution, and reproduction in any medium, provided the original work is properly cited.

\section{Introduction}

Consider the following problem of minimizing a convex separable logarithmic function subject to a strictly convex inequality constraint and bounded variables:

(CSL)

$$
\min \left\{c(\mathbf{x}) \equiv \sum_{j \in J} c_{j}\left(x_{j}\right) \equiv \sum_{j \in J}\left(-s_{j} \ln m_{j} x_{j}\right)\right\}
$$

subject to

$$
\begin{gathered}
\sum_{j \in J} d_{j}\left(x_{j}\right) \equiv \sum_{j \in J} d_{j} x_{j}^{p} \leq \alpha, \\
a_{j} \leq x_{j} \leq b_{j}, \quad j \in J,
\end{gathered}
$$

where $s_{j}>0, m_{j}>0, x_{j}>0, d_{j}^{\prime}\left(x_{j}\right)>0, j \in J, p \geq 1, \mathbf{x}=\left(x_{j}\right)_{j \in J}$, and $J \stackrel{\text { def }}{=}\{1, \ldots, n\}$. 
Since $c_{j}^{\prime \prime}\left(x_{j}\right)=s_{j} / x_{j}^{2}>0$, then $c_{j}\left(x_{j}\right), j \in J$, are convex functions defined for $x_{j}>0$, $m_{j}>0, j \in J$, and since $c_{j}^{\prime}\left(x_{j}\right)=-s_{j} / x_{j}<0$ under the assumptions, then functions $c_{j}\left(x_{j}\right)$, $j \in J$, are decreasing.

As it is known, $d_{j}\left(x_{j}\right)=d_{j} x_{j}^{p}, j \in J$, are convex functions with $d_{j} \geq 0, p \geq 1$ and $x_{j} \geq 0$, and strictly convex functions for $d_{j}>0, p>1$ and $x_{j}>0$. Since $d_{j}^{\prime}\left(x_{j}\right)=p d_{j} x_{j}^{p-1}$ and since $d_{j} \geq 0, p \geq 1$, then the requirement $d_{j}^{\prime}\left(x_{j}\right)>0$ becomes $d_{j}>0, x_{j}>0, j \in J$. In particular, since $x_{j}>0, j \in J$, then $a_{j}>0, b_{j}>0, j \in J$.

Similarly, consider the problem of minimizing a convex separable logarithmic function subject to a linear equality constraint and bounded variables:

(CSLE)

$$
\min \left\{c(\mathbf{x}) \equiv \sum_{j \in J} c_{j}\left(x_{j}\right) \equiv \sum_{j \in J}\left(-s_{j} \ln \left(1+m_{j} x_{j}\right)\right)\right\}
$$

subject to

$$
\begin{gathered}
\sum_{j \in J} d_{j} x_{j}=\alpha, \\
a_{j} \leq x_{j} \leq b_{j}, \quad j \in J,
\end{gathered}
$$

where $s_{j}>0, m_{j}>0, d_{j}>0, x_{j}>-1 / m_{j}, j \in J$. Using that $c_{j}^{\prime \prime}\left(x_{j}\right)=s_{j} m_{j}^{2} /(1+$ $\left.m_{j} x_{j}\right)^{2}>0$, it follows that $c_{j}\left(x_{j}\right)$ are strictly convex functions. Since $c_{j}^{\prime}\left(x_{j}\right)=$ $-s_{j} m_{j} /\left(1+m_{j} x_{j}\right)<0$ under the assumptions, then functions $c_{j}\left(x_{j}\right), j \in J$, are decreasing.

Problems (CSL) and (CSLE) are convex separable programming problems because the objective functions and constraint functions are convex (or strictly convex), and separable, that is, these functions can be expressed as the sums of single-variable functions.

It turns out that some problems, arising in production planning and scheduling, in allocation of resources $[2,6,7,14]$, in decision making $[2,7,10,12,14]$, in the theory of search, in subgradient optimization, in facility location $[10,12,13]$, and so forth, can be described mathematically by using problems like (CSL) and (CSLE), defined by (1.1)(1.3) and (1.4)-(1.6), respectively. That is why, in order to solve such practical problems, we need some results and methods for solving (CSL) and (CSLE).

Problems like (CSL) and (CSLE) are subject of intensive study. Related problems and methods for them are considered, for example, in [1-14].

Algorithms for resource allocation problems are proposed in [2, 6, 7, 14]. Algorithms for facility location problems are suggested in [10, 12], and so forth. Singly constrained quadratic programs with bounded variables are considered in $[3,5]$. Some separable programs are considered and methods for solving them are suggested in [11-13], and so forth.

Theory of nonlinear programming and, in particular, convex programming, is considered, for example, in $[8,9]$. Numerical methods for solving optimization problems are widely discussed, for example, in $[1,4]$. 
This paper is devoted to development of new efficient polynomial algorithms for solving problems (CSL) and (CSLE). The paper is organized as follows. In Section 2, characterization theorems (necessary and sufficient conditions) for the optimal solutions to the considered problems are proved. In Section 3, new algorithms of polynomial complexity are suggested and their convergence is proved. In Section 4, we consider some theoretical and numerical aspects of implementation of the algorithms and give some extensions of both characterization theorems and algorithms. In Section 5, we present results of some numerical experiments.

\section{Characterization theorems}

2.1. Problem (CSL). First consider problem (CSL) defined by (1.1)-(1.3).

Suppose that following assumptions are satisfied.

(1.a) $a_{j} \leq b_{j}$ for all $j \in J$. If $a_{k}=b_{k}$ for some $k \in J$ then the value $x_{k}:=a_{k}=b_{k}$ is determined a priori.

(1.b) $\sum_{j \in J} d_{j} a_{j}^{p} \leq \alpha$. Otherwise the constraints (1.2)-(1.3) are inconsistent and $X^{\leq}=\varnothing$ where $X^{\leq}$is defined by (1.2)-(1.3). In addition to this assumption we suppose that $\alpha \leq \sum_{j \in J} d_{j} b_{j}^{p}$ in some cases which are specified below.

The Lagrangian for problem (CSL) is

$$
L(\mathbf{x}, \mathbf{u}, \mathbf{v}, \lambda)=-\sum_{j \in J} s_{j} \ln m_{j} x_{j}+\lambda\left(\sum_{j \in J} d_{j} x_{j}^{p}-\alpha\right)+\sum_{j \in J} u_{j}\left(a_{j}-x_{j}\right)+\sum_{j \in J} v_{j}\left(x_{j}-b_{j}\right),
$$

where $\lambda \in \mathbb{R}_{+}^{1} ; \mathbf{u}, \mathbf{v} \in \mathbb{R}_{+}^{n}$, and $\mathbb{R}_{+}^{n}$ consists of all vectors with $n$ real nonnegative components.

The Karush-Kuhn-Tucker (KKT) necessary and sufficient optimality conditions for the minimum solution $\mathbf{x}^{*}=\left(x_{j}^{*}\right)_{j \in J}$ are

$$
\begin{gathered}
-\frac{s_{j}}{x_{j}^{*}}+\lambda p d_{j}\left(x_{j}^{*}\right)^{p-1}-u_{j}+v_{j}=0, \quad j \in J, \\
u_{j}\left(a_{j}-x_{j}^{*}\right)=0, \quad j \in J, \\
v_{j}\left(x_{j}^{*}-b_{j}\right)=0, \quad j \in J, \\
\lambda\left(\sum_{j \in J} d_{j}\left(x_{j}^{*}\right)^{p}-\alpha\right)=0, \quad \lambda \in \mathbb{R}_{+}^{1}, \\
\sum_{j \in J} d_{j}\left(x_{j}^{*}\right)^{p} \leq \alpha, \\
a_{j} \leq x_{j}^{*} \leq b_{j}, \quad j \in J, \\
u_{j} \in \mathbb{R}_{+}^{1}, \quad v_{j} \in \mathbb{R}_{+}^{1}, \quad j \in J,
\end{gathered}
$$

where $\lambda, u_{j}, v_{j}, j \in J$, are the Lagrange multipliers associated with the constraints (1.2), $a_{j} \leq x_{j}, x_{j} \leq b_{j}, j \in J$, respectively. If $a_{j}=-\infty$ or $b_{j}=+\infty$ for some $j$, we do not consider the corresponding condition (2.3) [(2.4)] and Lagrange multiplier $u_{j}\left[v_{j}\right.$, resp.] 
According to conditions (2.2)-(2.8), $\lambda \geq 0, u_{j} \geq 0, v_{j} \geq 0, j \in J$, and complementary conditions (2.3), (2.4), (2.5) must be satisfied. In order to find $x_{j}^{*}, j \in J$, from system (2.2)-(2.8), we have to consider all possible cases for $\lambda, u_{j}, v_{j}$ : all $\lambda, u_{j}, v_{j}$ equal to 0 ; all $\lambda$, $u_{j}, v_{j}$ different from 0 ; some of them equal to 0 , and some of them different from 0 . The number of these cases is $2^{2 n+1}$, where $2 n+1$ is the number of all $\lambda, u_{j}, v_{j}, j \in J,|J|=n$. This is an enormous number of cases, especially for large-scale problems. For example, when $n=1500$, we have $2^{3001} \approx 10^{900}$ cases. Moreover, in each case we have to solve a large-scale system of (nonlinear) equations in $x_{j}^{*}, \lambda, u_{j}, v_{j}, j \in J$. Therefore the direct application of KKT theorem, using explicit enumeration of all possible cases, for solving large-scale problems of the considered form, would not give a result and we need efficient methods to solve the problems under consideration.

The following Theorem 2.1 gives a characterization of the optimal solution to problem (CSL). Its proof, of course, is based on the KKT theorem. As we will see in Section 5, by using Theorem 2.1 and the algorithm based on it, we can solve problem (CSL) with $n=1500$ variables for a hundredth of a second on a personal computer.

Theorem 2.1 (characterization of the optimal solution to problem (CSL)). A feasible solution $\mathbf{x}^{*}=\left(x_{j}^{*}\right)_{j \in J} \in X^{\leq}$, where $X^{\leq}$is defined by (1.2)-(1.3), is the optimal solution to problem (CSL) if and only if there exists some $\lambda \in \mathbb{R}_{+}^{1}$ such that

$$
\begin{aligned}
& x_{j}^{*}=a_{j}, \quad j \in J_{a}^{\lambda} \stackrel{\text { def }}{=}\left\{j \in J: \lambda \geq \frac{s_{j}}{p d_{j} a_{j}^{p}}\right\}, \\
& x_{j}^{*}=b_{j}, \quad j \in J_{b}^{\lambda} \stackrel{\text { def }}{=}\left\{j \in J: \lambda \leq \frac{s_{j}}{p d_{j} b_{j}^{p}}\right\}, \\
& x_{j}^{*}=\sqrt[p]{\frac{s_{j}}{p \lambda d_{j}}}, \quad j \in J^{\lambda} \stackrel{\text { def }}{=}\left\{j \in J: \frac{s_{j}}{p d_{j} b_{j}^{p}}<\lambda<\frac{s_{j}}{p d_{j} a_{j}^{p}}\right\} .
\end{aligned}
$$

We will show below that $\lambda>0$ strictly, so that the expressions of $x_{j}^{*}, j \in J^{\lambda}$, in (2.11) (especially expressions under the radical sign) are correct.

Proof

Necessity. Let $\mathbf{x}^{*}=\left(x_{j}^{*}\right)_{j \in J}$ be the optimal solution to (CSL). Then there exist constants $\lambda, u_{j}, v_{j}, j \in J$, such that KKT conditions (2.2)-(2.8) are satisfied. Consider both possible cases for $\lambda$.

(1) Let $\lambda>0$. Then system (2.2)-(2.8) becomes (2.2), (2.3), (2.4), (2.7), (2.8) and

$$
\sum_{j \in J} d_{j}\left(x_{j}^{*}\right)^{p}=\alpha
$$

that is, the inequality constraint (1.2) is satisfied with an equality for $x_{j}^{*}, j \in J$, in this case.

(a) If $x_{j}^{*}=a_{j}$ then $u_{j} \geq 0$, and $v_{j}=0$ according to (2.4). Therefore (2.2) implies $-s_{j} / x_{j}^{*}=u_{j}-\lambda p d_{j}\left(x_{j}^{*}\right)^{p-1} \geq-\lambda p d_{j}\left(x_{j}^{*}\right)^{p-1}$. Since $d_{j}>0, x_{j}^{*}>0, j \in J, p \geq 1$ 
then

$$
\lambda \geq \frac{s_{j}}{p d_{j}\left(x_{j}^{*}\right)^{p}} \equiv \frac{s_{j}}{p d_{j} a_{j}^{p}} .
$$

(b) If $x_{j}^{*}=b_{j}$ then $u_{j}=0$ according to (2.3), and $v_{j} \geq 0$. Therefore (2.2) implies $-s_{j} / x_{j}^{*}=-v_{j}-\lambda p d_{j}\left(x_{j}^{*}\right)^{p-1} \leq-\lambda p d_{j}\left(x_{j}^{*}\right)^{p-1}$. Hence

$$
\lambda \leq \frac{s_{j}}{p d_{j}\left(x_{j}^{*}\right)^{p}} \equiv \frac{s_{j}}{p d_{j} b_{j}^{p}} .
$$

(c) If $a_{j}<x_{j}^{*}<b_{j}$ then $u_{j}=v_{j}=0$ according to (2.3) and (2.4). Therefore (2.2) implies $s_{j} / x_{j}^{*}=\lambda p d_{j}\left(x_{j}^{*}\right)^{p-1}$. Hence $\lambda=s_{j} / p d_{j}\left(x_{j}^{*}\right)^{p}$, and $x_{j}^{*}=\sqrt[p]{s_{j} / p \lambda d_{j}}$. Since $s_{j}>0, d_{j}>0, x_{j}^{*}>0, j \in J, p \geq 1, \lambda>0$ by the assumption and $b_{j}>x_{j}^{*}$, $x_{j}^{*}>a_{j}$, it follows that $\lambda=s_{j} / p d_{j}\left(x_{j}^{*}\right)^{p}<s_{j} / p d_{j} a_{j}^{p}, \lambda=s_{j} / p d_{j}\left(x_{j}^{*}\right)^{p}>s_{j} / p d_{j} b_{j}^{p}$, that is,

$$
\frac{s_{j}}{p d_{j} b_{j}^{p}}<\lambda<\frac{s_{j}}{p d_{j} a_{j}^{p}} .
$$

(2) Let $\lambda=0$. Then system (2.2)-(2.8) becomes

$$
-\frac{s_{j}}{x_{j}^{*}}-u_{j}+v_{j}=0, \quad j \in J, \quad(2.3),(2.4),(2.6),(2.7),(2.8) .
$$

(a) If $x_{j}^{*}=a_{j}$ then $u_{j} \geq 0, v_{j}=0$. Therefore $-s_{j} / a_{j} \equiv-s_{j} / x_{j}^{*}=u_{j} \geq 0$. Multiplying both sides of this inequality by $-1 / p d_{j} a_{j}^{p-1}$ ( $<0$ by the assumption), we obtain

$$
\frac{s_{j}}{p d_{j} a_{j}^{p}} \leq 0 \equiv \lambda
$$

Since $s_{j}>0, d_{j}>0, a_{j}>0, j \in J, p \geq 1$, this case is impossible.

(b) If $x_{j}^{*}=b_{j}$ then $u_{j}=0, v_{j} \geq 0$. Therefore $-s_{j} / b_{j} \equiv-s_{j} / x_{j}^{*}=-v_{j} \leq 0$. Multiplying this inequality by $-1 / p d_{j} b_{j}^{p-1}<0$, we get

$$
\frac{s_{j}}{p d_{j} b_{j}^{p}} \geq 0 \equiv \lambda
$$

(c) If $a_{j}<x_{j}^{*}<b_{j}$ then $u_{j}=v_{j}=0$. Therefore $-s_{j} / x_{j}^{*}=0$, and since $s_{j}>0, x_{j}^{*}>0$, $j \in J$, then this case is impossible.

To describe cases (a), (b), (c) for both (1) and (2), it is convenient to introduce the index sets $J_{a}^{\lambda}, J_{b}^{\lambda}, J^{\lambda}$ defined by (2.9), (2.10), and (2.11), respectively. It is obvious that $J_{a}^{\lambda} \cup J_{b}^{\lambda} \cup J^{\lambda}=J$. The "necessity" part is proved. 
Sufficiency. Conversely, let $\mathbf{x}^{*} \in X^{\leq}$and components of $\mathbf{x}^{*}$ satisfy (2.9), (2.10), and (2.11), where $\lambda \in \mathbb{R}_{+}^{1}$.

(1) Let $\lambda>0$. Set:

$\lambda=\frac{s_{j}}{p d_{j}\left(x_{j}^{*}\right)^{p}} \quad(>0), \quad$ obtained from $\sum_{j \in J_{a}^{\lambda}} d_{j} a_{j}^{p}+\sum_{j \in J_{b}^{\lambda}} d_{j} b_{j}^{p}+\sum_{j \in J^{\lambda}} \frac{s_{j}}{p^{\lambda}}=\alpha ;$

$u_{j}=v_{j}=0 \quad$ for $j \in J^{\lambda}$;

$u_{j}=-\frac{s_{j}}{a_{j}}+\lambda p d_{j} a_{j}^{p-1} \quad\left(\geq 0\right.$ according to the definition of $\left.J_{a}^{\lambda}\right), \quad v_{j}=0 \quad$ for $j \in J_{a}^{\lambda}$;

$u_{j}=0, \quad v_{j}=\frac{s_{j}}{b_{j}}-\lambda p d_{j} b_{j}^{p-1} \quad\left(\geq 0\right.$ according to the definition of $\left.J_{b}^{\lambda}\right) \quad$ for $j \in J_{b}^{\lambda}$.

By using these expressions, it is easy to check that conditions (2.2), (2.3), (2.4), (2.5), (2.8) are satisfied; conditions (2.6) and (2.7) are also satisfied according to the assumption $\mathbf{x}^{*} \in X^{\leq}$.

(2) If $\lambda=0$ then $-s_{j} / x_{j}^{*}-u_{j}+v_{j}=0, j \in J^{\lambda}$ according to (2.2), and

$$
J^{\lambda=0}=\left\{j: \frac{s_{j}}{p d_{j} b_{j}^{p}}<0<\frac{s_{j}}{p d_{j} a_{j}^{p}}\right\} .
$$

Since $s_{j}>0, d_{j}>0, b_{j}>0, j \in J, p \geq 1$, then $s_{j} / p d_{j} b_{j}^{p}>0$, and $J^{\lambda=0}=\varnothing$. Similarly, $J_{a}^{\lambda=0}=\varnothing$. Therefore $J=J_{b}^{\lambda=0}$ for $\lambda=0$.

Set:

$$
u_{j}=0, \quad v_{j}=\frac{s_{j}}{b_{j}} \quad(>0) \quad \text { for } j \in J=J_{b}^{\lambda=0} .
$$

Obviously conditions (2.2), (2.3), (2.4), (2.8) are satisfied; conditions (2.6), (2.7) are also satisfied according to the assumption $\mathbf{x}^{*} \in X^{\leq}$, and condition (2.5) obviously is satisfied for $\lambda=0$.

In both cases (1) and (2) of the "sufficiency" part, $x_{j}^{*}, \lambda, u_{j}, v_{j}, j \in J$, satisfy KKT conditions (2.2)-(2.8) which are necessary and sufficient conditions for a feasible solution to be an optimal solution to a convex minimization problem. Therefore $\mathbf{x}^{*}$ is an optimal solution to problem (CSL), and since $c(\mathbf{x})$ is a strictly convex function, then this optimal solution is unique.

In view of the discussion above, the importance of Theorem 2.1 consists in the fact that it describes components of the optimal solution to problem (CSL) only through the Lagrange multiplier $\lambda$ associated with the inequality constraint (1.2). 
Since we do not know the optimal value of $\lambda$ from Theorem 2.1, we define an iterative process with respect to the Lagrange multiplier $\lambda$ and we prove convergence of this process in Section 3 (The algorithms).

According to Theorem 2.1, $\lambda \geq 0$. In fact, since $p \lambda d_{j}\left(x_{j}^{*}\right)^{p}=s_{j}$, where $d_{j}>0, x_{j}^{*}>0$, $s_{j}>0, j \in J, p \geq 1$, then $\lambda>0$. Since $s_{j} / p d_{j} a_{j}^{p}>0, s_{j} / p d_{j} b_{j}^{p}>0$ under the assumptions, then if $\lambda:=0$ at step (2) of Algorithm 3.1 below, obviously $J^{\lambda=0}=J_{a}^{\lambda=0}=\varnothing$ and it would not be necessary to compute $x_{j}^{*}, j \in J^{0}$, using (2.11) for $\lambda=0$, where $\lambda$ is involved in the denominator of expression (2.11) for $x_{j}^{*}$. An analogous remark is also valid for problem (CSLE).

Using $d_{j}>0, s_{j}>0,0<a_{j} \leq b_{j}, j \in J$, and $p \geq 1$, it follows that

$$
u b_{j} \stackrel{\text { def }}{=} \frac{s_{j}}{p d_{j} b_{j}^{p}} \leq \frac{s_{j}}{p d_{j} a_{j}^{p}} \stackrel{\text { def }}{=} l a_{j}, \quad j \in J
$$

for the expressions by which we define the sets $J_{a}^{\lambda}, J_{b}^{\lambda}, J^{\lambda}$.

The problem how to ensure a feasible solution to problem (CSL), which is an assumption of Theorem 2.1, is discussed in Section 3.3.

2.2. Problem (CSLE). Consider the problem of minimizing a convex separable logarithmic function subject to a linear equality constraint and box constraints (CSLE) (1.4)(1.6).

\section{Assumptions:}

(2.a) $a_{j} \leq b_{j}$ for all $j \in J$.

(2.b) $\sum_{j \in J} d_{j} a_{j} \leq \alpha \leq \sum_{j \in J} d_{j} b_{j}$. Otherwise the constraints (1.5)-(1.6) are inconsistent and the feasible region $X^{L}$, defined by (1.5)-(1.6), is empty.

The KKT conditions for problem (CSLE) are

$$
\begin{gathered}
-\frac{s_{j} m_{j}}{1+m_{j} x_{j}^{*}}+\lambda d_{j}-u_{j}+v_{j}=0, \quad j \in J, \lambda \in \mathbb{R}^{1}, \\
u_{j}\left(a_{j}-x_{j}^{*}\right)=0, \quad j \in J, \\
v_{j}\left(x_{j}^{*}-b_{j}\right)=0, \quad j \in J, \\
\sum_{j \in J} d_{j} x_{j}^{*}=\alpha, \\
a_{j} \leq x_{j}^{*} \leq b_{j}, \quad j \in J, \\
u_{j} \in \mathbb{R}_{+}^{1}, \quad v_{j} \in \mathbb{R}_{+}^{1}, \quad j \in J .
\end{gathered}
$$

In this case, the following Theorem 2.2, which is similar to Theorem 2.1, holds true. Theorem 2.2 (characterization of the optimal solution to problem (CSLE)). A feasible solution $\mathbf{x}^{*}=\left(x_{j}^{*}\right)_{j \in J} \in X^{L}$, where $X^{L}$ is defined by (1.5)-(1.6), is the optimal solution to 
8 Minimizing a convex separable logarithmic function

problem (CSLE) if and only if there exists some $\lambda \in \mathbb{R}^{1}$ such that

$$
\begin{aligned}
& x_{j}^{*}=a_{j}, \quad j \in J_{a}^{\lambda} \stackrel{\text { def }}{=}\left\{j \in J: \lambda \geq \frac{s_{j} m_{j}}{d_{j}\left(1+m_{j} a_{j}\right)}\right\}, \\
& x_{j}^{*}=b_{j}, \quad j \in J_{b}^{\lambda} \stackrel{\text { def }}{=}\left\{j \in J: \lambda \leq \frac{s_{j} m_{j}}{d_{j}\left(1+m_{j} b_{j}\right)}\right\}, \\
& x_{j}^{*}=\frac{s_{j}}{\lambda d_{j}}-\frac{1}{m_{j}}, \quad j \in J^{\lambda} \stackrel{\text { def }}{=}\left\{j \in J: \frac{s_{j} m_{j}}{d_{j}\left(1+m_{j} b_{j}\right)}<\lambda<\frac{s_{j} m_{j}}{d_{j}\left(1+m_{j} a_{j}\right)}\right\} .
\end{aligned}
$$

It can be shown that $\lambda>0$ strictly, so that the expressions of $x_{j}^{*}, j \in J^{\lambda}$, in (2.26) are correct.

The proof of Theorem 2.2 is omitted because it is similar to that of Theorem 2.1.

\section{The algorithms}

3.1. Analysis of the optimal solution to problem (CSL). Before the formal statement of the algorithm for problem (CSL), we discuss some properties of its optimal solution.

Using (2.9), (2.10) and (2.11), condition (2.5) can be written as follows

$$
\lambda\left(\sum_{j \in J_{a}^{\lambda}} d_{j} a_{j}^{p}+\sum_{j \in J_{b}^{\lambda}} d_{j} b_{j}^{p}+\frac{1}{p \lambda} \sum_{j \in J^{\lambda}} s_{j}-\alpha\right)=0, \quad \lambda \geq 0 .
$$

Since the optimal solution $\mathbf{x}^{*}$ to problem (CSL) depends on $\lambda$, we consider components of $\mathbf{x}^{*}$ as functions of $\lambda$ for different $\lambda \in \mathbb{R}_{+}^{1}$ :

$$
x_{j}^{*}=x_{j}(\lambda)= \begin{cases}a_{j}, & j \in J_{a}^{\lambda} \\ b_{j}, & j \in J_{b}^{\lambda} \\ \sqrt[p]{\frac{s_{j}}{p \lambda d_{j}}}, & j \in J^{\lambda} .\end{cases}
$$

Functions $x_{j}(\lambda), j \in J$, are piecewise linear, monotonically nonincreasing, piecewise differentiable functions of $\lambda$ with two breakpoints at $\lambda=s_{j} / p d_{j} a_{j}^{p}$ and $\lambda=s_{j} / p d_{j} b_{j}^{p}$.

Let

$$
\delta(\lambda) \stackrel{\text { def }}{=} \sum_{j \in J_{a}^{\lambda}} d_{j} a_{j}^{p}+\sum_{j \in J_{b}^{\lambda}} d_{j} b_{j}^{p}+\frac{1}{p \lambda} \sum_{j \in J^{\lambda}} s_{j}-\alpha .
$$


If we differentiate $\delta(\lambda)$ with respect to $\lambda$, we get

$$
\delta^{\prime}(\lambda) \equiv-\frac{1}{p \lambda^{2}} \sum_{j \in J^{\lambda}} s_{j}<0,
$$

when $J^{\lambda} \neq \varnothing$, and $\delta^{\prime}(\lambda)=0$ when $J^{\lambda}=\varnothing$. Hence $\delta(\lambda)$ is a monotonically nonincreasing function of $\lambda \in \mathbb{R}_{+}^{1}$, and $\max _{\lambda \geq 0} \delta(\lambda)$ is attained at the minimum admissible value of $\lambda$, that is, at $\lambda=0$.

Case 1. Since $J^{\lambda=0}=J_{a}^{\lambda=0}=\varnothing$ then

$$
\delta(0)=\sum_{j \in J_{b}^{\lambda}} d_{j} b_{j}^{p}-\alpha
$$

according to (3.3), and $\delta(0) \geq 0$ in accordance with assumption (1.b). In order that (3.1) and (2.6) $\equiv(1.2)$ be satisfied, there exists some $\lambda^{*}>0$ such that $\delta\left(\lambda^{*}\right)=0$, that is,

$$
\sum_{j \in J} d_{j}\left(x_{j}^{*}\right)^{p}=\alpha
$$

which means that the inequality constraint (1.2) is satisfied with an equality for $\lambda^{*}$ in this case.

Case 2. The case $\delta(0)<0$ is impossible for problem (CSL) according to above consideration.

As we have seen, for the optimal value of $\lambda$ we have $\lambda \geq 0$ in all possible cases, as the KKT condition (2.5) requires. We have shown that in Case 1 we need an algorithm for finding $\lambda^{*}$, which satisfies the KKT conditions (2.2)-(2.8) and such that $\lambda^{*}$ satisfies (2.6) with an equality. In order that this be fulfilled, we have required $\alpha \leq \sum_{j \in J} d_{j} b_{j}^{p}$ in some cases in addition to the assumption $\sum_{j \in J} d_{j} a_{j}^{p} \leq \alpha$ (see assumption (1.b)). We have also used this assumption in the proof of Theorem 2.1, "sufficiency" part, when $\lambda>0$.

Using the equation $\delta(\lambda)=0$, where $\delta(\lambda)$ is defined by (3.3), we are able to obtain a closed form expression for $\lambda$ :

$$
\lambda=\frac{1}{p}\left(\alpha-\sum_{j \in J_{a}^{\lambda}} d_{j} a_{j}^{p}-\sum_{j \in J_{b}^{\lambda}} d_{j} b_{j}^{p}\right)^{-1} \sum_{j \in J^{\lambda}} s_{j},
$$

because $\delta^{\prime}(\lambda)<0$ according to (3.4) when $J^{\lambda} \neq \varnothing$ (it is important that $\delta^{\prime}(\lambda) \neq 0$ ). This expression of $\lambda$ is used in the algorithm suggested for problem (CSL). It turns out that without loss of generality we can assume that $\delta^{\prime}(\lambda) \neq 0$, that is, $\delta(\lambda)$ depends on $\lambda$, which means that $J^{\lambda} \neq \varnothing$.

At iteration $k$ of the implementation of algorithms, denote by $\lambda^{(k)}$ the value of the Lagrange multiplier associated with constraint (1.2) [(1.5), resp.], by $\alpha^{(k)}$ the right-hand side of (1.2) [(1.5), resp.]; by $J^{(k)}, J_{a}^{\lambda(k)}, J_{b}^{\lambda(k)}, J^{\lambda(k)}$ the current sets $J, J_{a}^{\lambda}, J_{b}^{\lambda}, J^{\lambda}$, respectively.

3.2. Algorithm 3.1 (for problem (CSL)). The following algorithm for solving problem (CSL) is based on Theorem 2.1, see Algorithm 3.1. 
(1) (Initialization) $J:=\{1, \ldots, n\}, k:=0, \alpha^{(0)}:=\alpha, n^{(0)}:=n, J^{(0)}:=J, J_{a}^{\lambda}:=\varnothing, J_{b}^{\lambda}:=$ $\varnothing$.

If $\sum_{j \in J} d_{j} a_{j}^{p} \leq \alpha \leq \sum_{j \in J} d_{j} b_{j}^{p}$, go to (2) else go to (9).

(2) $J^{\lambda(k)}:=J^{(k)}$. Calculate $\lambda^{(k)}$ by using the explicit expression (3.7) of $\lambda$. Go to (3).

(3) Construct the sets $J_{a}^{\lambda(k)}, J_{b}^{\lambda(k)}, J^{\lambda(k)}$ through (2.9), (2.10), (2.11) (with $j \in J^{(k)}$ instead of $j \in J)$ and find their cardinalities $\left|J_{a}^{\lambda(k)}\right|,\left|J_{b}^{\lambda(k)}\right|,\left|J^{\lambda(k)}\right|$, respectively. Go to (4).

(4) Calculate

$$
\delta\left(\lambda^{(k)}\right):=\sum_{j \in J_{a}^{\lambda(k)}} d_{j} a_{j}^{p}+\sum_{j \in J_{b}^{\lambda(k)}} d_{j} b_{j}^{p}+\frac{1}{p \lambda^{(k)}} \sum_{j \in J^{\lambda(k)}} s_{j}-\alpha^{(k)} .
$$

Go to (5).

(5) If $\delta\left(\lambda^{(k)}\right)=0$ or $J^{\lambda(k)}=\varnothing$ then $\lambda:=\lambda^{(k)}, J_{a}^{\lambda}:=J_{a}^{\lambda} \cup J_{a}^{\lambda(k)}, J_{b}^{\lambda}:=J_{b}^{\lambda} \cup J_{b}^{\lambda(k)}, J^{\lambda}:=$ $J^{\lambda(k)}$,

go to $(8)$

else if $\delta\left(\lambda^{(k)}\right)>0$ go to (6)

else if $\delta\left(\lambda^{(k)}\right)<0$ go to (7).

(6) $x_{j}^{*}:=a_{j}$ for $j \in J_{a}^{\lambda(k)}, \alpha^{(k+1)}:=\alpha^{(k)}-\sum_{j \in J_{a}^{\lambda(k)}} d_{j} a_{j}^{p}, J^{(k+1)}:=J^{(k)} \backslash J_{a}^{\lambda(k)}$, $n^{(k+1)}:=n^{(k)}-\left|J_{a}^{\lambda(k)}\right|, J_{a}^{\lambda}:=J_{a}^{\lambda} \cup J_{a}^{\lambda(k)}, k:=k+1$. Go to (2).

(7) $x_{j}^{*}:=b_{j}$ for $j \in J_{b}^{\lambda(k)}, \alpha^{(k+1)}:=\alpha^{(k)}-\sum_{j \in J_{b}^{\lambda(k)}} d_{j} b_{j}^{p}, J^{(k+1)}:=J^{(k)} \backslash J_{b}^{\lambda(k)}$, $n^{(k+1)}:=n^{(k)}-\left|J_{b}^{\lambda(k)}\right|, J_{b}^{\lambda}:=J_{b}^{\lambda} \cup J_{b}^{\lambda(k)}, k:=k+1$. Go to (2).

(8) $x_{j}^{*}:=a_{j}$ for $j \in J_{a}^{\lambda} ; x_{j}^{*}:=b_{j}$ for $j \in J_{b}^{\lambda} ; x_{j}^{*}:=\sqrt[p]{s_{j} / p \lambda d_{j}}$ for $j \in J^{\lambda}$. Go to (10).

(9) Problem (CSL) has no optimal solution because $X^{\leq}=\varnothing$ or there does not exist a $\lambda^{*}>0$ satisfying Theorem 2.1 .

(10) End.

Algorithm 3.1. (For problem (CSL)).

3.3. Convergence and complexity of Algorithm 3.1. The following Theorem 3.1 states convergence of Algorithm 3.1.

Theorem 3.1. Let $\lambda^{(k)}$ be the sequence generated by Algorithm 3.1. Then

(i) if $\delta\left(\lambda^{(k)}\right)>0$ then $\lambda^{(k)} \leq \lambda^{(k+1)}$;

(ii) if $\delta\left(\lambda^{(k)}\right)<0$ then $\lambda^{(k)} \geq \lambda^{(k+1)}$.

Proof. Denote by $x_{j}^{(k)}$ the components of $\mathbf{x}^{(k)}=\left(x_{j}\right)_{j \in J^{(k)}}$ at iteration $k$ of implementation of Algorithm 3.1.

(i) Let $\delta\left(\lambda^{(k)}\right)>0$. Using step (6) of Algorithm 3.1, which is performed when $\delta\left(\lambda^{(k)}\right)>0$, we get

$$
\sum_{j \in J^{\lambda(k+1)}} d_{j}\left(x_{j}^{(k)}\right)^{p} \equiv \sum_{j \in J^{(k+1)}} d_{j}\left(x_{j}^{(k)}\right)^{p}=\sum_{j \in J^{(k)} \backslash \bigcup_{a}^{\lambda(k)}} d_{j}\left(x_{j}^{(k)}\right)^{p}=\alpha^{(k)}-\sum_{j \in J_{a}^{\lambda(k)}} d_{j}\left(x_{j}^{(k)}\right)^{p} .
$$


Let $j \in J_{a}^{\lambda(k)}$. According to definition (2.9) of $J_{a}^{\lambda(k)}$ we have

$$
\frac{s_{j}}{p d_{j} a_{j}^{p}} \leq \lambda^{(k)}=\frac{s_{j}}{p d_{j}\left(x_{j}^{(k)}\right)^{p}} .
$$

Multiplying this inequality by $p d_{j} a_{j}^{p} / s_{j}>0$ we obtain $1 \leq a_{j}^{p} /\left(x_{j}^{(k)}\right)^{p}$. Therefore $x_{j}^{(k)} \leq a_{j}$ because $a_{j}>0, x_{j}^{(k)}>0$ and $p \geq 1$.

Using that $d_{j}>0, a_{j} \geq x_{j}^{(k)}, j \in J_{a}^{\lambda(k)}$, and step (6), from (3.9) we get

$$
\begin{aligned}
\sum_{j \in J^{\lambda(k+1)}} d_{j}\left(x_{j}^{(k)}\right)^{p} & =\alpha^{(k)}-\sum_{j \in J_{a}^{\lambda(k)}} d_{j}\left(x_{j}^{(k)}\right)^{p} \\
& \geq \alpha^{(k)}-\sum_{j \in J_{a}^{\lambda(k)}} d_{j} a_{j}^{p}=\alpha^{(k+1)}=\sum_{j \in J^{\lambda(k+1)}} d_{j}\left(x_{j}^{(k+1)}\right)^{p} .
\end{aligned}
$$

Since $d_{j}>0, j \in J$, then there exists at least one $j_{0} \in J^{\lambda(k+1)}$ such that $x_{j_{0}}^{(k)} \geq x_{j_{0}}^{(k+1)}$. Then

$$
\lambda^{(k)}=\frac{s_{j_{0}}}{p d_{j_{0}}\left(x_{j_{0}}^{(k)}\right)^{p}} \leq \frac{s_{j_{0}}}{p d_{j_{0}}\left(x_{j_{0}}^{(k+1)}\right)^{p}}=\lambda^{(k+1)} .
$$

We have used that the relationship between $\lambda^{(k)}$ and $x_{j}^{(k)}$ is given by (2.11) for $j \in J^{\lambda(k)}$ according to step (2) of Algorithm 3.1, and $\lambda^{(k)} \geq 0, d_{j}>0, s_{j}>0, j \in J$, and $p \geq 1$.

The proof of part (ii) is omitted because it is similar to that of part (i).

Consider the feasibility of $\mathbf{x}^{*}=\left(x_{j}^{*}\right)_{j \in J}$, generated by Algorithm 3.1.

Components $x_{j}^{*}=a_{j}, j \in J_{a}^{\lambda}$, and $x_{j}^{*}=b_{j}, j \in J_{b}^{\lambda}$, obviously satisfy (1.3). Using

$$
\frac{s_{j}}{p d_{j} b_{j}^{p}}<\lambda \equiv \frac{s_{j}}{p d_{j}\left(x_{j}^{*}\right)^{p}}<\frac{s_{j}}{p d_{j} a_{j}^{p}}, \quad j \in J^{\lambda}
$$

and $p \geq 1, d_{j}>0, s_{j}>0, a_{j}>0, b_{j}>0, j \in J$, it follows that $a_{j}<x_{j}^{*}<b_{j}$ for $j \in J^{\lambda}$. Hence all $x_{j}^{*}, j \in J$, satisfy $(1.3)$.

We have proved that if $\delta(0) \geq 0$ and $X^{\leq} \neq \varnothing$ where $X^{\leq}$is defined by (1.2)-(1.3), then there exists a $\lambda^{*} \geq 0$ such that $\delta\left(\lambda^{*}\right)=0$. Since at step (2) we calculate $\lambda^{(k)}$ from the equality $\sum_{j \in J^{\lambda(k)}} d_{j}\left(x_{j}^{(k)}\right)^{p}=\alpha^{(k)}$ for each $k$, then (1.2) is satisfied with an equality in this case. Otherwise, the case $\delta(0)<0$ is impossible (see Case 2 above). 
Therefore, Algorithm 3.1 generates $\mathbf{x}^{*}$ which is feasible for problem (CSL), which is an assumption of Theorem 2.1.

Remark 3.2. Theorem 3.1, definitions of $J_{a}^{\lambda}(2.9), J_{b}^{\lambda}$ (2.10) and $J^{\lambda}$ (2.11), and steps (6), (7) and (8) of Algorithm 3.1 allow us to assert that $J_{a}^{\lambda(k)} \subseteq J_{a}^{\lambda(k+1)}, J_{b}^{\lambda(k)} \subseteq J_{b}^{\lambda(k+1)}$, and $J^{\lambda(k)} \supseteq J^{\lambda(k+1)}$. This means that if $j$ belongs to current set $J_{a}^{\lambda(k)}$, then $j$ belongs to the next index set $J_{a}^{\lambda(k+1)}$ and, therefore, to the optimal index set $J_{a}^{\lambda}$; the same holds true about the sets $J_{b}^{\lambda(k)}$ and $J_{b}^{\lambda}$. Therefore $\lambda^{(k)}$ converges to the optimal $\lambda$ of Theorem 2.1 , and $J_{a}^{\lambda(k)}, J_{b}^{\lambda(k)}$, $J^{\lambda(k)}$ "converge" to the optimal index sets $J_{a}^{\lambda}, J_{b}^{\lambda}, J^{\lambda}$, respectively. This means that calculation of $\lambda$, operations $x_{j}^{*}:=a_{j}, j \in J_{a}^{\lambda(k)}$ (step (6)), $x_{j}^{*}:=b_{j}, j \in J_{b}^{\lambda(k)}$ (step (7)), and the construction of $J_{a}^{\lambda}, J_{b}^{\lambda}, J^{\lambda}$ are in accordance with Theorem 2.1.

At each iteration Algorithm 3.1 determines the value of at least one variable (steps (6), (7), (8)) and at each iteration we solve a problem of the form (CSL) but of less dimension (steps (2), (3), (4), (5), (6), (7)). Therefore Algorithm 3.1 is finite and it converges with at most $n=|J|$ iterations, that is, the iteration complexity of Algorithm 3.1 is $O(n)$.

Step (1) (initialization and checking whether $X^{\leq}$is empty) takes time $O(n)$. The calculation of $\lambda^{(k)}$ requires constant time (step (2)). Step (3) takes $O(n)$ time because of the construction of $J_{a}^{\lambda(k)}, J_{b}^{\lambda(k)}, J^{\lambda(k)}$. Step (4) also requires $O(n)$ time and step (5) requires constant time. Each of steps (6), (7) and (8) takes time which is bounded by $O(n)$, because at these steps we assign some of $x_{j}$ the final value, and since the number of all $x_{j}$ 's is $n$, then steps (6), (7) and (8) take time $\mathcal{O}(n)$. Hence Algorithm 3.1 has $\mathscr{O}\left(n^{2}\right)$ running time and it belongs to the class of strongly polynomially bounded algorithms.

Computational experiments show that the number of iterations of the algorithm performance is not only at most $n$, but it is much, much less than $n$ for large $n$. In fact, this number does not depend on $n$ but only on the three index sets defined by (2.9), (2.10), (2.11). In practice, Algorithm 3.1 has $\mathcal{O}(n)$ running time.

3.4. Algorithm 3.2 (for problem (CSLE)) and its convergence. After analysis of the optimal solution to problem (CSLE), similar to that to problem (CSL), we suggest the following algorithm for solving problem (CSLE).

To avoid a possible "endless loop" in programming Algorithms 3.1 and 3.2, the criterion of step (5) to go to step (8) at iteration $k$ usually is not $\delta\left(\lambda^{(k)}\right)=0$, but $\delta\left(\lambda^{(k)}\right) \in$ $[-\varepsilon, \varepsilon]$, where $\varepsilon>0$ is some (given or chosen) tolerance value up to which the equality $\delta\left(\lambda^{*}\right)=0$ may (for Algorithm 3.1) or must (for Algorithm 3.2) be satisfied.

A theorem analogous to Theorem 3.1 holds for Algorithm 3.2, which guarantees the "convergence" of $\lambda^{(k)}, J^{\lambda(k)}, J_{a}^{\lambda(k)}, J_{b}^{\lambda(k)}$ to the optimal $\lambda, J^{\lambda}, J_{a}^{\lambda}, J_{b}^{\lambda}$, respectively.

Theorem 3.3. Let $\lambda^{(k)}$ be the sequence generated by Algorithm 3.2. Then

(i) if $\delta\left(\lambda^{(k)}\right)>0$ then $\lambda^{(k)} \leq \lambda^{(k+1)}$;

(ii) if $\delta\left(\lambda^{(k)}\right)<0$ then $\lambda^{(k)} \geq \lambda^{(k+1)}$.

The proof of Theorem 3.3 is omitted because it is similar to that of Theorem 3.1.

It can be proved that Algorithm 3.2 has $\mathcal{O}\left(n^{2}\right)$ running time, and point $\mathbf{x}^{*}=\left(x_{j}^{*}\right)_{j \in J}$ generated by this algorithm is feasible for problem (CSLE), which is an assumption of Theorem 2.2. 
(1) (Initialization) $J:=\{1, \ldots, n\}, k:=0, \alpha^{(0)}:=\alpha, n^{(0)}:=n, J^{(0)}:=J, J_{a}^{\lambda}:=\varnothing, J_{b}^{\lambda}:=$ $\varnothing$.

If $\sum_{j \in J} d_{j} a_{j} \leq \alpha \leq \sum_{j \in J} d_{j} b_{j}$, go to (2) else go to (9).

(2) $J^{\lambda(k)}:=J^{(k)}$. Calculate $\lambda^{(k)}$ by using the explicit expression

$$
\lambda^{(k)}=\left(\alpha^{(k)}+\sum_{j \in J^{\lambda(k)}} \frac{d_{j}}{m_{j}}\right)^{-1} \sum_{j \in J^{\lambda(k)}} s_{j} .
$$

Go to (3).

(3) Construct the sets $J_{a}^{\lambda(k)}, J_{b}^{\lambda(k)}, J^{\lambda(k)}$ through (2.24), (2.25), (2.26) (with $j \in J^{(k)}$ instead of $j \in J)$ and find their cardinalities $\left|J_{a}^{\lambda(k)}\right|,\left|J_{b}^{\lambda(k)}\right|,\left|J^{\lambda(k)}\right|$. Go to (4).

(4) Calculate

$$
\delta\left(\lambda^{(k)}\right):=\sum_{j \in J_{a}^{\lambda(k)}} d_{j} a_{j}+\sum_{j \in J_{b}^{\lambda(k)}} d_{j} b_{j}+\frac{1}{\lambda^{(k)}} \sum_{j \in J^{\lambda(k)}} s_{j}-\sum_{j \in J^{\lambda(k)}} \frac{d_{j}}{m_{j}}-\alpha^{(k)} .
$$

Go to $(5)$.

(5) If $\delta\left(\lambda^{(k)}\right)=0$ or $J^{\lambda(k)}=\varnothing$ then $\lambda:=\lambda^{(k)}, J_{a}^{\lambda}:=J_{a}^{\lambda} \cup J_{a}^{\lambda(k)}, J_{b}^{\lambda}:=J_{b}^{\lambda} \cup J_{b}^{\lambda(k)}, J^{\lambda}:=$ $J^{\lambda(k)}$,

go to $(8)$

else if $\delta\left(\lambda^{(k)}\right)>0$ go to $(6)$

else if $\delta\left(\lambda^{(k)}\right)<0$ go to (7).

(6) $x_{j}^{*}:=a_{j}$ for $j \in J_{a}^{\lambda(k)}, \alpha^{(k+1)}:=\alpha^{(k)}-\sum_{j \in J_{a}^{\lambda(k)}} d_{j} a_{j}, J^{(k+1)}:=J^{(k)} \backslash J_{a}^{\lambda(k)}$, $n^{(k+1)}:=n^{(k)}-\left|J_{a}^{\lambda(k)}\right|, J_{a}^{\lambda}:=J_{a}^{\lambda} \cup J_{a}^{\lambda(k)}, k:=k+1$. Go to (2).

(7) $x_{j}^{*}:=b_{j}$ for $j \in J_{b}^{\lambda(k)}, \alpha^{(k+1)}:=\alpha^{(k)}-\sum_{j \in J_{b}^{\lambda(k)}} d_{j} b_{j}, J^{(k+1)}:=J^{(k)} \backslash J_{b}^{\lambda(k)}$, $n^{(k+1)}:=n^{(k)}-\left|J_{b}^{\lambda(k)}\right|, J_{b}^{\lambda}:=J_{b}^{\lambda} \cup J_{b}^{\lambda(k)}, k:=k+1$. Go to (2).

(8) $x_{j}^{*}:=a_{j}$ for $j \in J_{a}^{\lambda} ; x_{j}^{*}:=b_{j}$ for $j \in J_{b}^{\lambda} ; x_{j}^{*}:=s_{j} / \lambda d_{j}-1 / m_{j}$ for $j \in J^{\lambda}$. Go to (10).

(9) Problem (CSLE) has no optimal solution because the feasible set $X^{L}$, defined by (1.5)-(1.6), is empty.

(10) End.

Algorithm 3.2. (For problem (CSLE)).

\section{Extensions}

4.1. Theoretical aspects. Up to now we required $d_{j}>0, j \in J$, in (1.2) and (1.5) of problems (CSL) and (CSLE), respectively. However, if it is allowed $d_{j}=0$ for some $j$ in problems (CSL) and (CSLE), then for such indices $j$ we cannot construct the expressions $s_{j} / p d_{j} a_{j}^{p}$ and $s_{j} / p d_{j} b_{j}^{p}$ for problem (CSL), and $s_{j} m_{j} / d_{j}\left(1+m_{j} a_{j}\right)$ and $s_{j} m_{j} / d_{j}\left(1+m_{j} b_{j}\right)$ for problem (CSLE), by means of which we define sets $J_{a}^{\lambda}, J_{b}^{\lambda}, J^{\lambda}$ for the corresponding problem. In such cases, $x_{j}$ 's are not involved in (1.2) [in (1.5), resp.] for such indices $j$. 
14 Minimizing a convex separable logarithmic function

It turns out that we can avoid this difficulty and solve problems (CSL) and (CSLE) with $d_{j}=0$ for some $j$ 's.

Denote

$$
Z 0=\left\{j \in J: d_{j}=0\right\}
$$

Here " 0 " means the "computer zero." In particular, when $J=Z 0$ and $\alpha=0$, then $X^{\leq}\left(X^{L}\right.$, resp.) is defined only by (1.3) (by (1.6), resp.).

Theorem 4.1 (characterization of the optimal solution to problem (CSL): an extended version). Problem (CSL) can be decomposed into two subproblems: (CSL1) for $j \in Z 0$ and (CSL2) for $j \in J \backslash Z 0$.

The optimal solution to (CSL1) is

$$
x_{j}^{*}=b_{j}, \quad j \in Z 0,
$$

that is, subproblem (CSL1) itself is decomposed into $n_{0} \equiv|Z 0|$ independent problems. The optimal solution to (CSL2) is given by (2.9), (2.10), (2.11) with $J:=J \backslash Z 0$.

Proof

Necessity. Let $\mathbf{x}^{*}=\left(x_{j}^{*}\right)_{j \in J}$ be the optimal solution to (CSL).

(1) Let $j \in Z 0$, that is, $d_{j}=0$ for this $j$. The KKT conditions are

$$
-\frac{s_{j}}{x_{j}^{*}}-u_{j}+v_{j}=0, j \in Z 0, \quad(2.3)-(2.8)
$$

(a) If $x_{j}^{*}=a_{j}$, then $u_{j} \geq 0, v_{j}=0$. Using (4.3), it follows that $-s_{j} / x_{j}^{*}=u_{j} \geq 0$, which is impossible because $s_{j}>0, x_{j}^{*}>0$.

(b) If $x_{j}^{*}=b_{j}$, then $u_{j}=0, v_{j} \geq 0$. Therefore $-s_{j} / x_{j}^{*}=-v_{j} \leq 0$, which is always satisfied for $s_{j}>0, x_{j}^{*}>0$.

(c) If $a_{j}<x_{j}^{*}<b_{j}$, then $u_{j}=v_{j}=0$. Therefore $-s_{j} / x_{j}^{*}=0$, that is, $s_{j}=0$, which is impossible according to the assumption $s_{j}>0$.

As we have observed, only case (b) is possible for $j \in Z 0$.

(2) Components of the optimal solution to (CSL2) are obtained by using the same approach as that of the proof of "necessity" part of Theorem 2.1, but with the reduced index set $J:=J \backslash Z 0$.

Sufficiency. Conversely, let $\mathbf{x}^{*} \in X^{\leq}$and components of $\mathbf{x}^{*}$ satisfy: (4.2) for $j \in Z 0$, and (2.9), (2.10), (2.11) for $J:=J \backslash Z 0$. Set:

$$
u_{j}=0, \quad v_{j}=\frac{s_{j}}{b_{j}} \quad(>0) \quad \text { for } j \in Z 0
$$


If $\lambda>0$, set:

$$
\begin{aligned}
& \lambda=\frac{s_{j}}{p d_{j}\left(x_{j}^{*}\right)^{p}}=\lambda\left(\mathbf{x}^{*}\right) \quad(>0) \quad \text { from }(2.11) ; \\
& u_{j}=v_{j}=0 \quad \text { for } a_{j}<x_{j}^{*}<b_{j}, j \in J \backslash Z 0 ; \\
& u_{j}=-\frac{s_{j}}{a_{j}}+\lambda p d_{j} a_{j}^{p-1} \quad(\geq 0), \quad v_{j}=0 \quad \text { for } x_{j}^{*}=a_{j}, j \in J \backslash Z 0 ; \\
& u_{j}=0, \quad v_{j}=\frac{s_{j}}{b_{j}}-\lambda p d_{j} b_{j}^{p-1} \quad(\geq 0) \quad \text { for } x_{j}^{*}=b_{j}, j \in J \backslash Z 0 .
\end{aligned}
$$

If $\lambda=0$, set:

$$
u_{j}=0, \quad v_{j}=\frac{s_{j}}{b_{j}} \quad(>0) \quad \text { for } j \in J \backslash Z 0 .
$$

As in the proof of Theorem 2.1, $J_{a}^{\lambda=0}=J^{\lambda=0}=\varnothing$.

It can be verified that $\mathbf{x}^{*}, \lambda, u_{j}, v_{j}, j \in J$, satisfy the KKT conditions (2.2)-(2.8). Then $\mathbf{x}^{*}$ with components: (4.2) for $j \in Z 0$, and (2.9), (2.10), (2.11) with $J:=J \backslash Z 0$, is the optimal solution to problem $(\mathrm{CSL})=(\mathrm{CSL} 1) \cup(\mathrm{CSL} 2)$.

An analogous result holds for problem (CSLE).

Theorem 4.2 (characterization of the optimal solution to problem (CSLE): an extended version). Problem (CSLE) can be decomposed into two subproblems: (CSLE1) for $j \in Z 0$ and (CSLE2) for $j \in J \backslash Z 0$.

The optimal solution to (CSLE1) is also given by (4.2). The optimal solution to (CSLE2) is given by (2.24), (2.25), (2.26) with $J:=J \backslash Z 0$.

The proof of Theorem 4.2 is omitted because it repeats in part the proofs of Theorems 2.1 and 4.1 .

Thus, with the use of Theorems 4.1 and 4.2 we can express components of the optimal solutions to problems (CSL) and (CSLE) without the necessity of constructing the expressions $s_{j} / p d_{j} a_{j}^{p}, s_{j} / p d_{j} b_{j}^{p}, s_{j} m_{j} / d_{j}\left(1+m_{j} a_{j}\right)$, and $s_{j} m_{j} / d_{j}\left(1+m_{j} b_{j}\right)$ with $d_{j}=0$.

4.2. Computational aspects. Algorithms 3.1 and 3.2 are also applicable in cases when $a_{j}=-\infty$ for some $j \in J$ and/or $b_{j}=\infty$ for some $j \in J$. However, if we use the computer values of $-\infty$ and $+\infty$ at step (1) of the algorithms to check whether the corresponding feasible region is empty or nonempty, and at step (3) in the expressions $s_{j} / p d_{j} x_{j}^{p}$ and $s_{j} m_{j} / d_{j}\left(1+m_{j} x_{j}\right)$ with $x_{j}=-\infty$ and/or $x_{j}=+\infty$, by means of which we construct sets $J_{a}^{\lambda}, J_{b}^{\lambda}, J^{\lambda}$, this could sometimes lead to arithmetic overflow. If we use other values of $-\infty$ and $+\infty$ with smaller absolute values than those of the computer values of $-\infty$ and $+\infty$, it would lead to inconvenience and dependence on the data of the particular problems. To avoid these difficulties and to take into account the above discussion, it is convenient to do the following. 
Construct the sets of indices:

$$
\begin{aligned}
S V N & =\left\{j \in J \backslash Z 0: a_{j}>-\infty, b_{j}<+\infty\right\}, \\
S V 1 & =\left\{j \in J \backslash Z 0: a_{j}>-\infty, b_{j}=+\infty\right\}, \\
S V 2 & =\left\{j \in J \backslash Z 0: a_{j}=-\infty, b_{j}<+\infty\right\}, \\
S V & =\left\{j \in J \backslash Z 0: a_{j}=-\infty, b_{j}=+\infty\right\} .
\end{aligned}
$$

It is obvious that $Z 0 \cup S V \cup S V 1 \cup S V 2 \cup S V N=J$, that is, the set $J \backslash Z 0$ is partitioned into the four subsets $S V N, S V 1, S V 2, S V$, defined above.

When programming the algorithms, we use computer values of $-\infty$ and $+\infty$ for constructing the sets $S V N, S V 1, S V 2, S V$.

In order to construct the sets $J_{a}^{\lambda}, J_{b}^{\lambda}$, $J^{\lambda}$ without the necessity of calculating the values $s_{j} / p d_{j} x_{j}^{p}$ with $x_{j}=-\infty$ or $+\infty$ for problem (CSL), except for the sets $J, Z 0, S V, S V 1, S V 2$, $S V N$, we need some subsidiary sets defined as follows.

For $S V N$ :

$$
\begin{aligned}
& J^{\lambda S V N}=\left\{j \in S V N: \frac{s_{j}}{p d_{j} b_{j}^{p}}<\lambda<\frac{s_{j}}{p d_{j} a_{j}^{p}}\right\}, \\
& J_{a}^{\lambda S V N}=\left\{j \in S V N: \lambda \geq \frac{s_{j}}{p d_{j} a_{j}^{p}}\right\}, \\
& J_{b}^{\lambda S V N}=\left\{j \in S V N: \lambda \leq \frac{s_{j}}{p d_{j} b_{j}^{p}}\right\} ;
\end{aligned}
$$

for $S V 1$ :

$$
\begin{aligned}
& J^{\lambda S V 1}=\left\{j \in S V 1: \lambda<\frac{s_{j}}{p d_{j} a_{j}^{p}}\right\}, \\
& J_{a}^{\lambda S V 1}=\left\{j \in S V 1: \lambda \geq \frac{s_{j}}{p d_{j} a_{j}^{p}}\right\} ;
\end{aligned}
$$

for SV2:

$$
\begin{aligned}
& J^{\lambda S V 2}=\left\{j \in S V 2: \lambda>\frac{s_{j}}{p d_{j} b_{j}^{p}}\right\}, \\
& J_{b}^{\lambda S V 2}=\left\{j \in S V 2: \lambda \leq \frac{s_{j}}{p d_{j} b_{j}^{p}}\right\} ;
\end{aligned}
$$

for $S V$ :

$$
J^{\lambda S V}=S V .
$$


Step $(1)^{1}$ (Initialization) $J:=\{1, \ldots, n\}, k:=0, \alpha^{(0)}:=\alpha, n^{(0)}:=n, J^{(0)}:=J, J_{a}^{\lambda}:=\varnothing$, $J_{b}^{\lambda}:=\varnothing$.

Construct the set $Z 0$. If $j \in Z 0$ then $x_{j}^{*}:=b_{j}$.

Set $J:=J \backslash Z 0, J^{(0)}:=J, n^{(0)}:=n-|Z 0|$.

Construct the sets $S V N, S V 1, S V 2, S V$.

If $S V N \cup S V 1=J$ then

if $\sum_{j \in J} d_{j} a_{j}^{p} \leq \alpha$ go to step (2)

else go to step (9) (feasible region $X^{\leq}$is empty)

else if $S V 2 \cup S V \neq \varnothing$ then

if $S V 2 \cup S V N=J$ then

if $\alpha \leq \sum_{j \in J} d_{j} b_{j}^{p}$ go to step (2)

else go to step (9) (there does not exist a $\lambda^{*}>0$ such that $\delta\left(\lambda^{*}\right)=0$ )

else if $S V 1 \cup S V \neq \varnothing$ go to step (2) (there exists a $\lambda^{*}>0$ such that $\delta\left(\lambda^{*}\right)=0$ ).

Step $(3)^{1}$. Construct the sets $J^{\lambda S V N}, J_{a}^{\lambda S V N}, J_{b}^{\lambda S V N}, J^{\lambda S V 1}, J_{a}^{\lambda S V 1}, J^{\lambda S V 2}, J_{b}^{\lambda S V 2}, J^{\lambda S V}$

(with $J^{(k)}$ instead of $J$ ).

Construct the sets $J_{a}^{\lambda(k)}, J_{b}^{\lambda(k)}, J^{\lambda(k)}$ by using (4.12) and find their cardinalities $\left|J_{a}^{\lambda(k)}\right|,\left|J_{b}^{\lambda(k)}\right|,\left|J^{\lambda(k)}\right|$, respectively. Go to step (4).

Algorithm 4.1. About Algorithm 3.1.

Then:

$$
\begin{gathered}
J^{\lambda}:=J^{\lambda S V N} \cup J^{\lambda S V 1} \cup J^{\lambda S V 2} \cup J^{\lambda S V}, \\
J_{a}^{\lambda}:=J_{a}^{\lambda S V N} \cup J_{a}^{\lambda S V 1}, \\
J_{b}^{\lambda}:=J_{b}^{\lambda S V N} \cup J_{b}^{\lambda S V 2} .
\end{gathered}
$$

We use the sets $J^{\lambda}, J_{a}^{\lambda}, J_{b}^{\lambda}$, defined by (4.12), as the corresponding sets with the same names in Algorithms 3.1 and 3.2.

With the use of results of this section, steps (1) and (3) of Algorithm 3.1 can be modified as follows, respectively, see Algorithm 4.1.

Similarly, we can define subsidiary index sets of the form (4.8)-(4.11) for problem (CSLE) as well, and modify steps (1) and (3) of Algorithm 3.2.

Modifications of the algorithms, connected with theoretical and computational aspects, do not influence upon their computational complexity, discussed in Section 3, because these modifications do not affect the "iterative" steps of algorithms.

\section{Computational experiments}

In this section, we present results of some numerical experiments, obtained by applying algorithms, suggested in this paper, to problems under consideration. The computations were performed on an Intel Pentium II Celeron Processor $466 \mathrm{MHz} / 128 \mathrm{MB}$ SDRAM 
Minimizing a convex separable logarithmic function

Table 5.1

\begin{tabular}{lcccc}
\hline Problem & \multicolumn{2}{c}{ (CSL) } & \multicolumn{2}{c}{ (CSLE) } \\
\hline Number of variables & $n=1200$ & $n=1500$ & $n=1200$ & $n=1500$ \\
Average number of iterations & 2.10 & 3.03 & 3.07 & 4.10 \\
Average run time (in seconds) & 0.06 & 0.067 & 0.00009 & 0.00011 \\
\hline
\end{tabular}

IBM PC compatible. Each problem was run 30 times. Coefficients $s_{j}>0, m_{j}>0, d_{j}>0$, $j \in J$, for problems (CSL) and (CSLE) were randomly generated, see Table 5.1.

When $n<1200$, the run time of the algorithms is so small that the timer does not recognize the corresponding value from its computer zero. In such cases the timer displays "0 seconds."

The effectiveness of algorithms for problems (CSL) and (CSLE) has been tested by many other examples. As we can observe, the (average) number of iterations is much less than the number of variables $n$ for large $n$.

We provide below the solution of two simple particular problems of the form (CLS) and (CLSE), respectively, obtained by using the approach suggested in this paper. The results are rounded to the fourth digit.

Example 5.1.

$$
\min \left\{c(\mathbf{x})=-\ln \left(2 x_{1}\right)-3 \ln x_{2}\right\}
$$

subject to

$$
x_{1}^{2}+2 x_{2}^{2} \leq 10, \quad 1 \leq x_{1} \leq 3, \quad 1 \leq x_{2} \leq 5 .
$$

The optimal solution, obtained by Algorithm 3.1, is

$$
\begin{gathered}
\mathbf{x}^{*}=\left(x_{1}^{*}, x_{2}^{*}\right)=(1.5811,1.9365), \\
c_{\min }=c\left(\mathbf{x}^{*}\right)=-3.1339 .
\end{gathered}
$$

Number of iterations: 1 .

Example 5.2.

$$
\min \left\{c(\mathbf{x})=-2 \ln \left(1+2 x_{1}\right)-\ln \left(1+3 x_{2}\right)\right\}
$$

subject to

$$
x_{1}+2 x_{2}=10, \quad 1 \leq x_{1} \leq 3, \quad 1 \leq x_{2} \leq 5 .
$$

The optimal solution, obtained by Algorithm 3.2, is

$$
\begin{gathered}
\mathbf{x}^{*}=\left(x_{1}^{*}, x_{2}^{*}\right)=(3.0,3.5), \\
c_{\min }=c\left(\mathbf{x}^{*}\right)=-5.2149 .
\end{gathered}
$$

Number of iterations: 2 . 


\section{Conclusions}

In this paper, we propose an efficient method for solving convex separable minimization problems with logarithmic objective function subject to convex inequality constraint or linear equality constraint, and box constraints.

This approach could be continued and generalized for minimizing arbitrary convex separable objective functions over the same feasible regions.

\section{Acknowledgment}

The author is grateful to the editor and referees for their comments and suggestions.

\section{References}

[1] M. S. Bazaraa, H. D. Sherali, and C. M. Shetty, Nonlinear Programming. Theory and Algorithms, 2nd ed., John Wiley \& Sons, New York, 1993.

[2] G. R. Bitran and A. C. Hax, Disaggregation and resource allocation using convex knapsack problems with bounded variables, Management Science 27 (1981), no. 4, 431-441.

[3] J.-P. Dussault, J. A. Ferland, and B. Lemaire, Convex quadratic programming with one constraint and bounded variables, Mathematical Programming 36 (1986), no. 1, 90-104.

[4] R. Fletcher, Practical Methods of Optimization, 2nd ed., John Wiley \& Sons, Chichester, 1987.

[5] R. Helgason, J. Kennington, and H. Lall, A polynomially bounded algorithm for a singly constrained quadratic program, Mathematical Programming 18 (1980), no. 3, 338-343.

[6] N. Katoh, T. Ibaraki, and H. Mine, A polynomial time algorithm for the resource allocation problem with a convex objective function, Journal of the Operations Research Society 30 (1979), no. 5, $449-455$.

[7] H. Luss and S. K. Gupta, Allocation of effort resources among competing activities, Operations Research 23 (1975), no. 2, 360-366.

[8] O. L. Mangasarian, Nonlinear Programming, 2nd ed., Classics in Applied Mathematics, vol. 10, SIAM, Pennsylvania, 1994.

[9] R. T. Rockafellar, Convex Analysis, Princeton Landmarks in Mathematics, Princeton University Press, New Jersey, 1997.

[10] S. M. Stefanov, On the implementation of stochastic quasigradient methods to some facility location problems, Yugoslav Journal of Operations Research 10 (2000), no. 2, 235-256.

[11] Convex separable minimization subject to bounded variables, Computational Optimization and Applications 18 (2001), no. 1, 27-48.

[12] Separable Programming. Theory and Methods, Applied Optimization, vol. 53, Kluwer Academic Publishers, Dordrecht, 2001.

[13] - Convex separable minimization problems with a linear constraint and bounded variables, International Journal of Mathematics and Mathematical Sciences 2005 (2005), no. 9, 13391363.

[14] P. H. Zipkin, Simple ranking methods for allocation of one resource, Management Science 26 (1980), no. 1, 34-43.

Stefan M. Stefanov: Department of Mathematics, South-West University "Neofit Rilski,"

2700 Blagoevgrad, Bulgaria

E-mail address: stefm@aix.swu.bg 


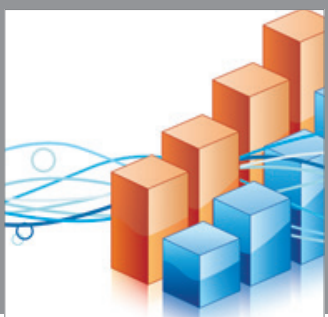

Advances in

Operations Research

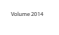

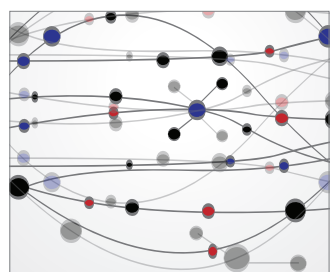

\section{The Scientific} World Journal
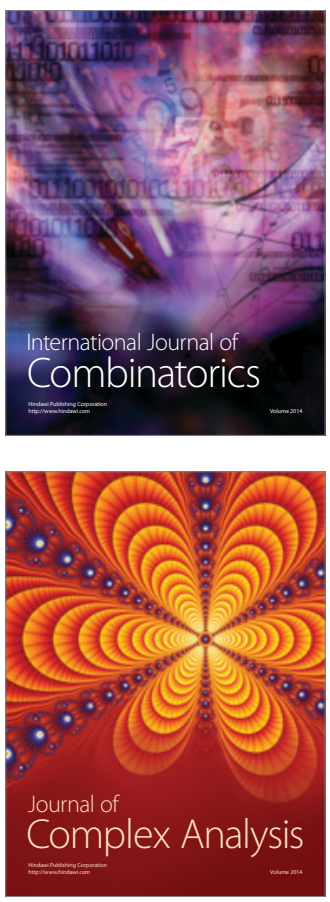

International Journal of

Mathematics and

Mathematical

Sciences
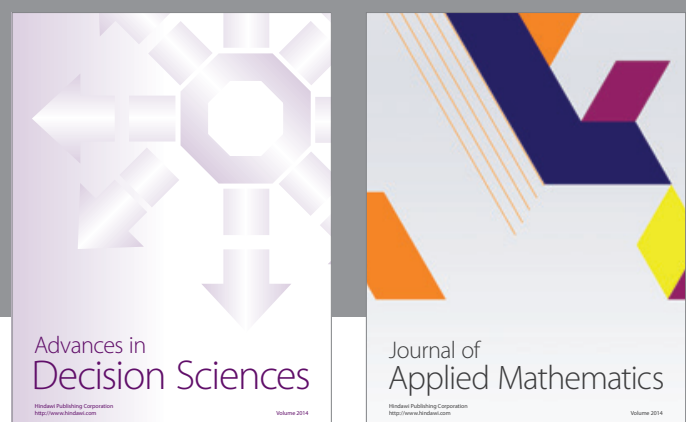

Journal of

Applied Mathematics
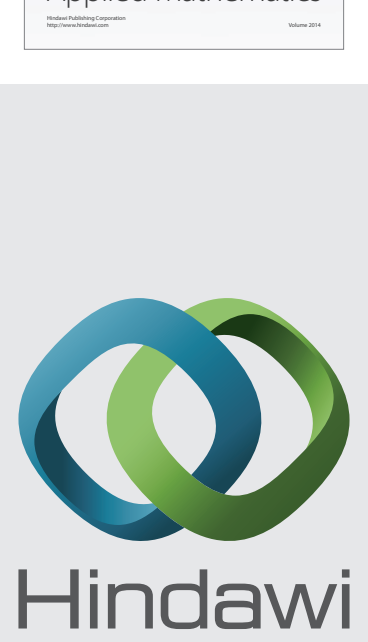

Submit your manuscripts at http://www.hindawi.com
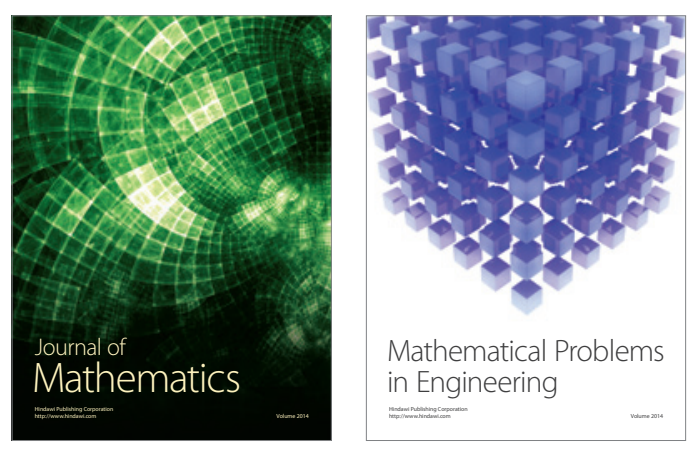

Mathematical Problems in Engineering
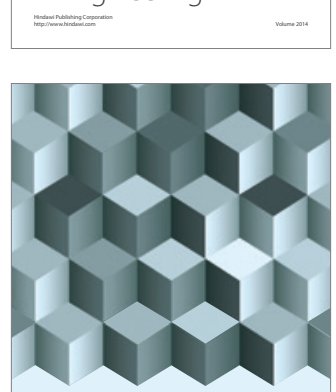

Journal of

Function Spaces
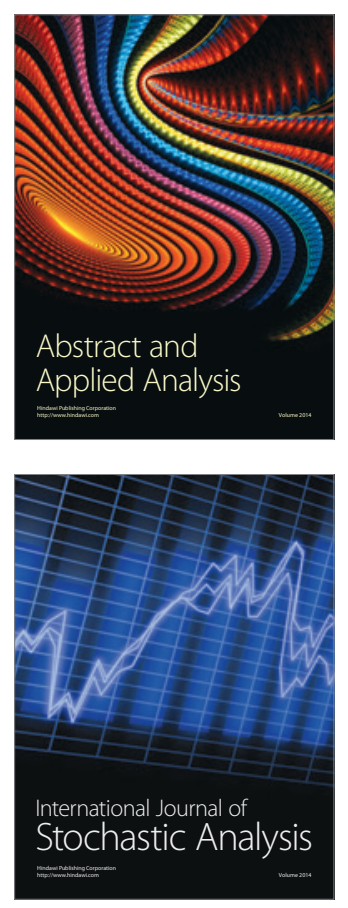

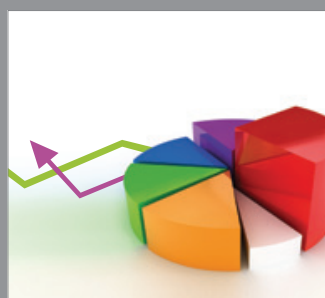

ournal of

Probability and Statistics

Promensencen
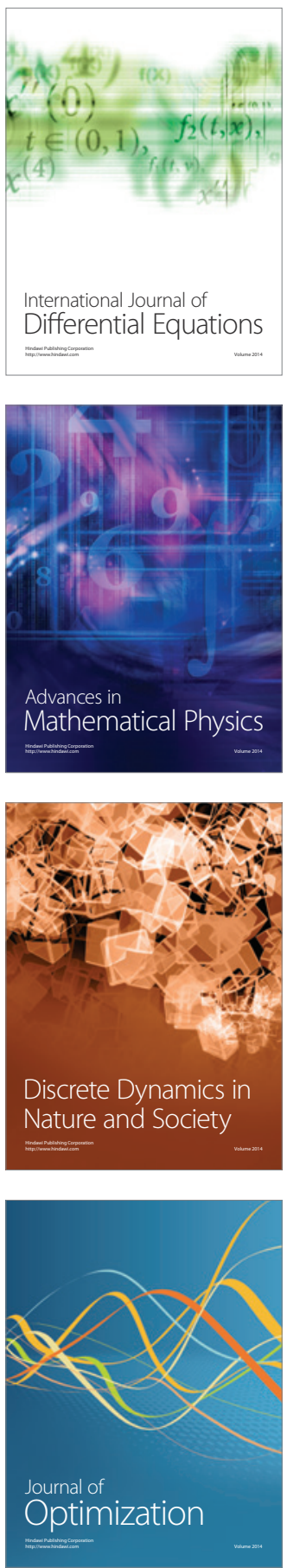